\title{
NATURSCHUTZ UND VERFASSUNGSRECHT
}

\section{ISSN 1727-3781}

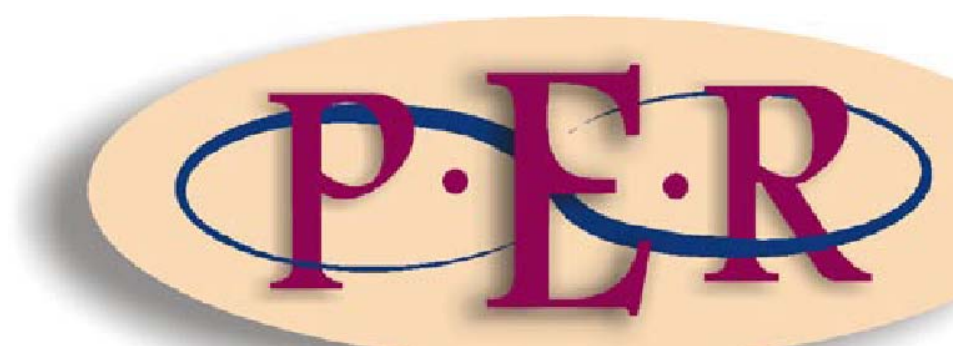

1999 VOLUME 2 No 1 


\section{NATURSCHUTZ UND VERFASSUNGSRECHT}

Prof Dr D Czybulka

\section{$1 \quad$ Einführung}

\subsection{Naturschutz und Schutz der natürlichen Lebensgrundlagen in der Rechtssprache}

In den Verfassungen des Bundes und der Länder wird der Begriff "Naturschutz" -anders als in den Naturschutzgesetzen, dem sogenannten "einfachen" Recht - außer in der Kompetenzvorschrift des Art. 75 Abs. 1 Nr. 3 des Grundgesetzes (GG) nicht ausdrücklich verwendet. Der Naturschutz wird im Verfassungsrecht zumeist als "Schutz der natürlichen Lebensgrundlagen" angesprochen. ${ }^{1}$ Im juristischen Schrifttum werden die "natürlichen Lebensgrundlagen" (als Schutzgut des Art. 20a GG) abgegrenzt von sozialen, ökonomischen, kulturellen oder technischen Lebensgrundlagen. ${ }^{2}$ Natürliche Lebensgrundlagen sind die Güter, die auch $\S 2$ des Gesetzes über die Umweltverträglichkeitsprüfung (UVPG) beschreibt, also Pflanzen, Tiere, Boden, Wasser, Luft, Klima, Landschaft und die Wechselbeziehungen zwischen innen. "Natürlich" ist vom Ansatz her alles, was nicht auf menschlicher Her-vorbringung beruht. Da es wilde oder unberührte Natur in Mitteleuropa kaum mehr gibt, bezieht sich Naturschutz auch auf die von Menschen kultivierten Landschaften. Der ökologisch zu begreifende Naturschutz wird als Teil des Umweltschutzes, in der Folge davon auch das Naturschutzrecht als Teil des Umweltschutzrechtes begriffen. Beides müßte vom Begrifflichen, vom Schutzgegenstand und vom geeigneten Instrumentarium her genauer gefaßt werden. Der normativ vermittelte Naturschutz unterscheidet sich von der Zielrichtung, aber auch in gravierenden Einzelfragen erheblich vom -zumeist technischen - Umweltschutz. Dies betrifft zunächst die ethischen Vorgaben für den Umgang des Menschen mit der Natur. ${ }^{4}$ Daneben sind wichtige Unterschiede im

1 Vgl Art 20a GG.

$2 \quad$ Murswiek Kommentierung zu Art 20a GG Rn 28.

3 Peters 1995 NVWZ 555.

$4 \quad$ Niesslein Naturschutz und Industriegesellschaft 45 ff. 
Steuerungsansatz, dem rechtlichen Instrumentarium und der Regelungstechnik auszumachen.

\subsection{Naturschutz in der Rechtsordnung}

Naturschutz wird in Deutschland, in Europa und den meisten Staaten der Erde auch mit Hilfe der Rechtsordnung umgesetzt beziehungsweise abgesichert. Die Bedeutung des Rechts für den Schutz und die Erhaltung der Natur sollte allerdings nicht überschätzt werden. Die Naturgesetze selbst sind rechtlicher Regelung nicht zugänglich, lediglich das menschliche Verhalten gegenüber der Natur, ${ }^{5}$ und letzteres auch nur in Teilbereichen. Wichtiger für einen effektiven Naturschutz dürften insoweit wirtschaftliche Faktoren und Entwicklungen sowie Einstellung und Erziehung der Bevölkerung gegenüber der Natur sein. ${ }^{6}$ Rechtsvorschriften haben unterschiedlichen Rang. In der nationalen Rechtsordnung steht das Verfassungsrecht an der Spitze der Normenhierarchie. In der Bundesrepublik Deutschland gibt es Verfassungsrecht auf zwei unterschiedlichen staatlichen Ebenen, nämlich der Ebene des Bundes und der 16 Länder. Die Verfassung des Bundes ist das Grundgesetz für die Bundesrepublik Deutschland, das seit 1994 die Staatszielbestimmung des Art. 20a GG zum Schutze der natürlichen Lebensgrundlagen enthält. Daneben enthalten die Verfassungen der 16 Bundesländer zumeist entsprechende oder sogar weitergehende Vorschriften. Hierbei ist zu beachten, daß etwa entgegenstehendes Landesverfassungsrecht gemäß Art. 31 GG ("Bundesrecht bricht Landesrecht") auch von einer untergesetzlichen Norm des Bundes (zum Bespiel einer Rechtsverordnung) außer Kraft gesetzt werden könnte. Das Verfassungsrecht selbst umfaßt verschiedene Normkategorien, die zwar alle Verfassungsrang besitzen, aber unterschiedlich wirken. Vereinfacht kann man hier zwischen Kompetenzvorschriften, Staatszielbestimmungen und den Grundrechten unterscheiden. Als weitere, umstrittene Kategorie wird die der Grundpflichten genannt. Wohl am schwierigsten zu beantworten ist in diesem Zusammenhang die verfassungstheoretische und -praktische Frage nach dem Verhältnis des (verrechtlichten) Naturschutzes zu den Grundrechten und zur "Würde des Menschen", die gemäß Art. 1 Abs. 1 GG unantastbar ist. Durch die "Ewigkeitsgarantie" des Art. 79 Abs. 3

$5 \quad$ Czybulka 1988 NuR 214.

$6 \quad$ Czybulka 1997 AgrarR 306. 
$\mathrm{GG}^{7}$ ist der Schutz der Menschenwürde innerhalb der Verfassung auf den höchsten Rang gesetzt worden. Zwar ist auch bei dieser anthropozentrischen Sichtweise der Schluß nicht zwingend, die Natur solle von der Verfassung nur soweit geschützt werden, als sie als Resource für den Menschen schutzwürdig erscheine; gleichwohl gestaltet sich eine ökologisch orientierte Rechts- beziehungsweise Verfassungstheorie außerordentlich schwierig.

\section{$2 \quad$ Naturschutz im deutschen ${ }^{10}$ Verfassungsrecht}

\subsection{Kompetenzvorschriften}

Mit Kompetenzvorschriften wird die Verteilung staatlicher Aufgaben und Befugnisse auf die staatlichen Organe geregelt. Im Bundesstaat teilt die Verfassung insbesondere die Gesetzgebungsbefugnisse und die Verwaltungsbefugnisse zwischen Bund und Ländern auf. Die Bedeutung der Kompetenzvorschriften erschöpft sich nicht in dieser technischorganisatorischen Aufteilung, sondern die Verfassungsbestimmungen erheben zugleich die entsprechenden Staatsaktivitäten zu Staatsaufgaben, ohne allerdings konkrete Verpflichtungen zu begründen. Nach Art. 75 Abs. 1 Nr. 3 GG hat der Bund die Rahmengesetzgebungskompetenz für die Gesetzgebungsmaterie Naturschutz (und die Landschaftspflege). ${ }^{11}$ Nach der Systematik der Verfassung bedeutet dies, daß der Schwerpunkt der Gesetzgebungstätigkeit in diesem Bereich bei den Ländern liegen soll, weil Rahmenvorschriften des Bundes nach Art. 75 Abs. 2 GG nur in Einzelfällen in Einzelheiten gehende oder unmittelbar geltende Regelungen enthalten dürfen. Das geltende Naturschutzrecht wird gleichwohl maßgeblich vom Bundesnaturschutzgesetz (BNatSchG) bestimmt. Die Bedeutung der Rahmengesetze des Bundes ergibt sich nicht nur aus den unmittelbar geltenden Vorschriften (so gelten etwa die Ziele und

7 "Eine Änderung dieses Grundgesetzes, durch welche .... die in den Art 1 und 20 niedergelegten Grundsätze berührt werden, ist unzulässig".

$8 \quad$ Vgl die Argumente bei Heinz 1990 Der Staat 424.

9 Vgl weiterführend Nida-Rümelin und Von der Pfordten Ökologische Ethik.

10 Die Darstellung muß sich auf das deutsche Verfassungsrecht beschränken. Das Verfassungsrecht des Bundes ist insoweit in Europa nur von allenfalls durchschnittlichem Schutzniveau. Wichtig für das Naturschutzrecht werden zunehmend das Europäische Recht und das -allerdings oft wenig wirksame - Völkerrecht.

11 Das gleiche gilt für den "Wasserhaushalt" Art 75 Abs 1 Nr 4 GG. 
Grundsätze des Naturschutzes nach $\S \S$ 1, 2 BNatSchG und damit auch die Landwirtschaftsklausel, das sogenannte Agrarprivileg, unmittelbar ${ }^{12}$ ), sondern auch daraus, daß der Bundesgesetzgeber grundlegende Begriffsbestimmungen treffen kann, die vom Landesgesetzgeber dann wegen Art. 31 GG nicht modifiziert werden können. Dies betrifft etwa die in der Praxis sehr wichtige Eingriffs- und Ausgleichsregelung. Der Nachteil einer Rahmengesetzgebung liegt in der Schwerfälligkeit und Mehrstufigkeit des Gesetzgebungsverfahrens, was sich auch negativ bei der Umsetzung von Richtlinien des Europäischen Rechts (zum Beispiel der FFH-Richtlinie) und von internationalen Abkommen bemerkbar macht.

Die Verwaltungskompetenz im Bereich des Naturschutzes liegt nach der allgemeinen verfassungsrechtlichen Zuordnung ${ }^{13}$ bei den Ländern. Soweit die Landesnaturschutzgesetze vollzogen werden, ist dies ohnehin selbstverständlich. Bei den Ländern liegt auch in der Praxis der Schwerpunkt staatlicher Naturschutztätigkeit. Es verbleibt dem Bund ein (begrenzter) Bereich eigener Betätigung, insbesondere bei internationalen Aktivitäten, konzeptionellen Vorarbeiten nationaler und grenzüberschreitender Dimension (Nationalparke, Biosphärenreservate), im Bereich der Forschung und der Finanzierung gesamtstaatlich-repräsentativer Vorhaben.

\subsection{Staatszielbestimmungen}

\subsubsection{Definition der Staatszielbestimmung als verfassungsrechtliche Kategorie}

Während die ursprüngliche Fassung des Grundgesetzes nur Kompetenzbestimmungen zum Naturschutz enthielt, gibt es seit dem 42. Änderungsgesetz vom 27.10.1994 den Art. 20a GG als Staatszielbestimmung. Unter Staatszielbestimmungen versteht die Verfassungslehre bindende Bestimmungen (also keine bloßen Programmsätze) der Verfassung, die sich vor allem an die staatlichen Organe wenden und innen die fortdauernde Beachtung oder Erfüllung sachlich umschriebener Ziele vorschreiben. ${ }^{15}$ Diese werden ihrerseits abgegrenzt zu den sogenannten Staatsstrukturprinzipien des

\footnotetext{
12 Vgl § 4 S 3 BNatSchG.

13 Art 3083 ff GG.

14 Czybulka 1996 NuR 565-570.

15 Henneke 1995 NuR 330.
} 
Grundgesetzes, die in Art. 20 Abs. 1 bis 3 GG (und in Art. 28 GG, insoweit bindend für die Länder) festgelegt sind. Zu den Staatsstrukturprinzipien zählt etwa das demokratische Prinzip und das Prinzip der Gewaltenteilung. Staatszielbestimmungen vermitteln im Unterschied zu den Grundrechten dem einzelnen Bürger keine subjektiven Rechte (etwa durchsetzbare Abwehransprüche gegen staatliche Eingriffe in die Natur), sondern haben ausschließlich objektiv-rechtlichen Charakter. ${ }^{16}$ Die Nichteinhaltung der Staatszielbestimmung kann deshalb vom einzelnen Staatsbürger nur insoweit gerichtlich durchgesetzt werden, als ihm das sonstige öffentliche Recht in dieser Beziehung eigene subjektive Rechte vermittelt. Das ist gerade im Bereich des Naturschutzes sehr selten.

\title{
2.2.2 Die Diskussion um die Aufnahme einer Staatszielbestimmung zum Schutz der natürlichen Lebensgrundlagen im Grundgesetz
}

Der geltende Art. 20a des Grundgesetzes lautet:

\begin{abstract}
"Der Staat schützt auch in Verantwortung für die künftigen Generationen die natürlichen Lebensgrundlagen im Rahmen der verfassungsmäßigen Ordnung durch die Gesetzgebung und nach Maßgabe von Gesetz und Recht durch die vollziehende Gewalt und die Rechtsprechung."
\end{abstract}

Die umständliche Formulierung ist Ausdruck eines politischen Kompromisses. Die zahlreichen Zusätze zum sachlichen Kern "Der Staat schützt auch in Verantwortung für die künftigen Generationen die natürlichen Lebensgrundlagen" werden auch als "Angstklausel" bezeichnet, die nach dem Urteil namhafter Staatrechtslehrer überflüssig sind. ${ }^{17}$ Seit dem Anfang der Siebziger Jahre setzte in Deutschland ein Meinungsstreit ein, ob und wie dem Umweltschutzgedanken verfassungsrechtlich adäquat Rechnung zu tragen sei. Obwohl die seinerzeitige SPD/FDP-Koalition mit ihrem bereits 1970 eingeleiteten Versuch, die Rahmenzuständigkeit des Bundes für Naturschutz und Landschaftspflege in eine Vollkompetenz des Bundes umzuwandeln, scheiterte, führte dieser politische Vorstoß unter andere zu bedeutsamen Verfassungsänderungen in 
mehreren Bundesländern, so 1976 in Baden-Württemberg zu einer Aufnahme der "natürlichen Lebensgrundlagen" in die Staatszielbestimmung des Art. 86 der Landesverfassung. ${ }^{18} \quad$ Eine Reihe anderer Bundesländer folgte. Der Einigungsvertrag vom 31. August 1990 gab dann einen neuen Anstoß zur Verfassungsreform. In der verfassungspolitischen Diskussion ging es bei der Ausgestaltung des rechtlichen Schutzes um die Alternativen der Schaffung eines "Umweltgrundrechts", der Aufnahme einer Staatszielbestimmung oder der Beibehaltung des Status quo (ausführlich zur Entwicklung ${ }^{19}$ ). Frühzeitig wurde auch die Auffassung vertreten, die diskutierten Alternativen seien aus ökologischer Sicht ein Anachronismus, weil die Anthropozentrik im Grundgesetz hierbei völlig ungebrochen bliebe. ${ }^{20}$ Diesem Einwand ist später (unten 3.2) nachzugehen.

Befürworter der Einführung eines Umweltgrundrechts (in Kombination mit einer Staatszielbestimmung) waren im politischen Bereich DIE GRÜNEN. Danach sollte den Rechten des Menschen auf Leben und körperliche Unversehrtheit in Art. 2 Abs. 2 S. 1 GG das Recht auf ...

"... die Erhaltung seiner natürlichen Lebensgrundlagen und den Schutz vor erheblichen Beeinträchtigungen seiner natürlichen Umwelt"

... hinzugefügt werden. Außerdem erstreckte der Entwurf die Sozialpflichtigkeit des Eigentums $^{21}$ auf die "Erhaltung der natürlichen Lebensgrundlagen". ${ }^{22}$ Schließlich sollte Art. 20 Abs. 1 GG erheblich erweitert, die natürliche Umwelt auch "um ihrer selbst willen unter den besonderen Schutz des Staates" gestellt und im Konfliktfall zwischen ökonomischen Erfordernissen und ökologischen Belangen letzteren ein (relativer) Vorrang eingeräumt werden. Der Entwurf der SPD-Fraktion sah im wesentlichen die Einfügung eines Art. 20a mit folgendem Wortlaut vor:

18 Bereits 1971 war die SchwVerf in Art 24 septies zum "Schutz des Menschen und seiner natürlichen Umwelt gegen schädliche oder lästige Einwirkungen" um eine obligatorische Bundeskompetenz erweitert worden.

19 Tsai Umweltschutzpflicht des Staates $48 \mathrm{ff}$.

20 Bosselmann 1987 NuR 6.

21 Art14 Abs 2 GG. 
"Die natürlichen Lebensgrundlagen stehen unter dem besonderen Schutz des Staates."

Das Schutzgut müsse umfassend und ohne anthropozentrische Verengung formuliert werden. Diesen Vorschlag lehnten die seinerzeit CDU- oder CSU- geführten Länder ab, die einen Gesetzesvorbehalt verlangten. Außerdem könne einer Staatszielbestimmung Umweltschutz kein höherer Rang gegenüber anderen Verfassungswerten zukommen. ${ }^{24}$ Damit sind die Kernstreitpunkte (Anthropozentrik oder "Ökozentrik", besonderer Schutz beziehungsweise relatives Abwägungsgewicht, Gesetzesvorbehalt) angesprochen. Herausgekommen ist nach langen und kontroversen Beratungen in der Gemeinsamen Verfassungskommission die eingangs wiedergegebene Formulierung.

\subsubsection{Berücksichtigung des Naturschutzes in den Landesverfassungen}

Schon in den Landesverfassungen der unmittelbaren Nachkriegszeit, die diesbezüglich vor allem Programmsätze enthielten, war die Aufgabe des Schutzes von Natur und Landschaft nicht nur dem Staat, sondern auch den Selbstverwaltungskörperschaften, insbesondere also den Gemeinden und Gemeindeverbänden anheimgestellt. ${ }^{25}$ Daneben finden sich auch detailliertere Verfassungsbestimmungen, die heimatpflegerische oder ästhetische Gesichtspunkte stärker hervorheben. So bestimmte Art. 141 Abs. 2 der bayerischen Verfassung von 1946:

"Der deutsche Wald, kennzeichnende Orts- und Landschaftsbilder und die einheimischen Tier- und Pflanzenarten sind möglichst zu schonen und zu erhalten."

Eine Besonderheit stellte Art. 141 Abs. 3 S. 1 der bayerischen Verfassung dar. Er gab jedermann in einem Teilbereich ein Grundrecht auf "Naturgenuß":

22 BT-Drs 11/663. Nach hier vertretener Auffassung ist allerdings die "Ökologiepflichtigkeit" des (Grund)Eigentums geltendes Recht.

23 BT-Drs 11/10; dies war auch - bis auf eine stilistische Änderung - der Vorschlag der Kommission Verfassungsreform des Bundesrates im Bericht 1992, BR-Drs 360/92, Rn 132 ff.

24 Henneke 1995 NuR $326 \mathrm{f}$. 


\begin{abstract}
"Der Genuß der Naturschönheiten und die Erholung in der freien Natur, insbesondere das Betreten von Wald und Bergweide, das Befahren der Gewässer und die Aneignung wildwachsender Waldfrüchte in ortsüblichem Umfang ist jedermann gestattet."
\end{abstract}

Nach der anfänglichen Rechtsprechung des Bayerischen Verfassungsgerichtshofes wurde damit nicht nur den Grundstückseigentümern Duldungspflichten auferlegt, sondern Art. 141 Abs. 3 S. 1 vermittelte ein echtes, subjektives Abwehrrecht gegen rechtswidrige Beeinträchtigungen der freien Natur. ${ }^{26}$ Zwischenzeitlich ist das Recht in der Interpretation der Rechtsprechung geschrumpft auf ein "Recht auf Genuß der Natur in ihrem jeweiligen Bestand". ${ }^{27}$

In den Siebziger und Achtziger Jahren wurden viele Verfassungsbestimmungen der alten Bundesländer, die den Naturschutz beziehungsweise den Umweltschutz betreffen, geändert oder neu eingeführt. Regelmäßig handelte es sich dabei um Staatszielbestimmungen. ${ }^{28}$ Die Bedeutung dieser Verfassungsänderungen war jedoch gering, weil die rechtliche Wirkung sich nur im Landesrecht entfalten durfte. So war zum Beispiel die Interpretation der Landwirtschaftsklauseln im Naturschutzrecht in Richtung auf eine naturverträgliche Landwirtschaft verstellt, weil insoweit das Bundesrecht, insbesondere die §§ 1 Abs. 3; 8 Abs. 7; $20 f$ Abs. 3 BNatSchG vorgingen.

Die neuen Bundesländer haben von Beginn ihrer staatlichen Existenz an allesamt derartige Staatszielbestimmungen in ihre Landesverfassungen aufgenommen. ${ }^{29}$ Diese Staatszielbestimmungen sind zum Teil zusätzlich verfassungsrechtlich abgesichert, daneben finden sich ergänzende Inhalte. In der Verfassung des Freistaates Thüringen

25 Vgl zB BayVerf vom 2.12.1946, Art 141 I S 1 aF: "Die Denkmäler der Kunst, der Geschichte und der Kultur, die Landschaft und Naturdenkmale genießen öffentlichen Schutz und die Pflege des Staates, der Gemeinden und der Körperschaften des öffentlichen Rechts."

26 Hofmann $1988 \mathrm{JZ} 274$.

27 So schon der BayVerwGH mit Urteil vom 11.6.1975 (VGH nF 30, 65); diesem offenbar folgend BayVGH, Entscheidung vom 23.08.1985 BayVBI 1985 683, 684.

28 Vgl BWVerf Art 86; BayVerf Art 2 Abs 2 und 141; BremVerf Art 11a; HambVerf Präambel; HessVerf Art 26a; NdsVerf Art 1 II; NWVerf Art 29a; RhPfVerf Art 69; SaarlVerf Art 59a; SchlHVerf Art 7.

29 BerlVerf Art 21a; BdbgVerf Art 39, 40; MVVerf Art 12; SächsVerf Art 10; SachsAnhVerf Art 35; ThürVerf Art 31. 
etwa befaßt sich zunächst ein eigener Abschnitt der Verfassung mit "Natur und Umwelt"; zuvor wird in der Präambel der Wille bekundet, Natur und Umwelt zu bewahren und zu schützen. ${ }^{30}$ Verfassungsrechtlich ist verankert, daß Tiere "als Mitgeschöpfe und Lebewesen geachtet" werden. Während diese Regelung eher dem Tierschutz zuzurechnen ist, findet sich in Brandenburg die (auch auf Pflanzen bezogene) Ergänzung: "Art und artgerechter Lebensraum sind zu erhalten und zu schützen", ${ }^{32}$ eine etwa für die Erhaltung der Großtrappenbestände (Otis tarda) durchaus einschlägige Bestimmung. Ferner wird das durch das Europäische Recht erstmals eingeführte Recht des Bürgers auf "Umweltdaten" landesverfassungsrechtlich für erhobene Daten über die "natürliche Umwelt in seinem Lebensraum" verfassungsrechtlich verankert. In Mecklenburg-Vorpommern ist die Eingriffsund Ausgleichsregelung landesverfassungsrechtlich abgesichert. ${ }^{34}$ In Brandenburg und Sachsen ist die Verbandsklage verfassungsrechtlich verankert. ${ }^{35}$ Zweifellos am weitesten geht der Text der brandenburgischen Verfassung. Neben seiner Erwähnung in der Präambel und bei den "Grundsätzen der Verfassung" ${ }^{36}$ findet der Schutz der natürlichen Lebensgrundlagen eine ausführliche Regelung in Art. 38 bis 40 der Verfassung. Nach dem Wortlaut des Art. 39 Abs. 2 BdbgVerf ist der Schutz der Unversehrtheit (des Menschen) "vor Verletzungen und unzumutbaren Gefährdungen, die aus Veränderungen der natürlichen Lebensgrundlagen entstehen" als subjektiv-öffentliches (Abwehr-)Recht, möglicherweise als Grundrecht ausgestaltet (hierzu näher unten 3.3.1).

\subsection{Die aktuelle Bedeutung der Staatszielbestimmungen zum Schutz der natürlichen Lebensgrundlagen für Gesetzgebung, Verwaltung und Rechtsprechung}

Staatszielbestimmungen sind keine empirischen Beschreibungen, sondern haben normatives Gewicht. Das Staatsziel beansprucht als objektiv-rechtlicher Verfassungssatz

30 Verfassung des Freistaates Thüringen vom 25.10.1993 (GVBI 625), Präambel, Art 31-33.

31 ThürVerf Art

32; $\quad$ vgl auch BdbgVerf Art 39 Abs 3. 32 BdbgVerf Art 39 Abs 3.

33 ThürVerf Art 33; vgl auch BdbgVerf Art 39 Abs 7.

$34 \quad$ MVVerf Art 12 Abs 4.

$35 \quad$ Vgl BdbgVerf Art 39 Abs 8; SächsVerf Art 10 Abs 2.

36 BdbgVerf Art 2 Abs 1 vereint die Verpflichtung zum Schutz der natürlichen Umwelt (und der Kultur) in einer Bestimmung mit den bekannten Staatszielen des freiheitlichen, demokratischen und sozialen Rechtsstaats sowie der Zusammenarbeit mit anderen Völkern, insbesondere mit dem polnischen Nachbarn. Knapper, aber inhaltlich ganz ähnlich SachsAnhVerf Art 2 Abs 1. 
unmittelbare Geltung und Verbindlichkeit. ${ }^{37}$ Art. 20a GG ist -wie alle Staatszielbestimmungen - kein bloßer Gesetzgebungsauftrag, wenn sich auch Art. 20a GG nach Auffassung des Bundesverwaltungsgerichts "in erster Linie an den Gesetzgeber" wendet, den die Verpflichtung trifft, den in dieser Norm enthaltenen Gestaltungsauftrag umzusetzen. Darüber hinaus richtet sich die Staatszielbestimmung aber auch an die übrigen Gewalten, also auch an die Exekutive und an die Gerichte. Art. 20a GG könnte dann so gelesen werden: "Alle Staatsorgane sind verpflichtet, die natürlichen Lebensgrundlagen zu schützen". ${ }^{39}$ Staatszielbestimmungen sind aber insoweit "unvollkommen", als ihnen kein entsprechender Rechtsschutz der Bürger korrespondiert. Weil Staatszielbestimmungen als rein objektiv-rechtlich aufgefaßt werden, fehlt es bereits an der Klagebefugnis (vergleich §42 Abs. 2 VwGO) und damit an der Zulässigkeit einer Klage, wenn dem Bürger nicht in anderer Weise ein subjektives, öffentliches Recht eingeräumt ist, das möglicherweise verletzt ist. Die in der Mehrzahl der Bundesländer eingeführte Verbandsklage erklärt deshalb für anerkannte Umweltverbände in bestimmten Verfahren die Klage ausdrücklich für zulässig, obwohl eine (eigene) Rechtsverletzung nicht geltend gemacht werden kann.

\subsubsection{Staatszielbestimmungen als Auslegungshilfe für die Interpretation einfachen Rechts}

Durch die Staatszielbestimmung des Art. 20a GG beziehungsweise in beschränkterem Umfang der Parallelvorschriften der Landesverfassungen kann sich - zum Teil sogar maßgeblich -die Auslegung von "unbestimmten Rechtsbegriffen" in unterverfassungsrechtlichen Normen ändern. Soweit diese Normen Bezug nehmen auf die "natürlichen Lebensgrundlagen" und ihren Schutz, ist Art. 20a GG in seiner Funktion als verfassungsrechtlich angeordneter Auslegungsgrundsatz zu beachten. ${ }^{40}$ Diese Bezugnahme muß nicht unbedingt ausdrücklich erfolgen; vielmehr dürfte bei allen gesetzlichen Regelungen, die auf "öffentliche Belange" abstellen oder bei denen das

37 Statt aller Murswiek 1996 NVwZ 223.

38 BVerwG NJW 1995, 2648, 2649.

39 So ausdrücklich Murswiek 1996 NVWZ 223; ähnlich Tsai Umweltschutzpflicht des Staates 212 These 6.

40 So ausdrücklich Uhle 1996 UPR 55, 56; Murswiek Kommentierung zu Art 20a GG Rn 68. 
"öffentliche Interesse" relevant ist, das gleiche gelten. ${ }^{41}$ Das Bundesverwaltungsgericht hat eine derartige Auslegung beispiels-weise bei der Interpretation des Außenbereichsschutzes nach § 35 BauGB vorgenommen. ${ }^{42}$ Grundsätzlich gilt das Prinzip der "Einheit der Rechtsordnung", so daß Gesetze, die die Naturzerstörung fördern, heute "verfassungskonform" interpretiert werden müssen. Dies gilt etwa für die sogenannte "Rohstoffsicherungsklausel" im Bergrecht. Der Staat darf die Zerstörung der Umwelt jedenfalls nicht erleichtern oder fördern. Damit ist das aus dem Umweltrecht stammende Verursacherprinzip möglicherweise zum Verfassungsinhalt geworden. Umweltzerstörung kann auf Dauer keine "Gemeinlast" mehr sein. ${ }^{43}$ Die Übertragung des Verursacherprinzips auf das Naturschutzrecht fällt insoweit schwer, weil hier zunächst der Schutz und die Erhaltung der Natur im Vordergrund steht, nicht das Problem der Beseitigung (eventuell des Ausgleiches und des Ersatzes) von und der Kostenverteilung für Eingriffe, die letztlich die Natur zerstören.

\subsubsection{Die Staatszielbestimmung des Art. 20a GG als Ermessendirektive}

Die Staatszielbestimmung des Art. 20a kann auch als Ermessensdirektive wirken. Wenn der entscheidenden Behörde ein echter Ermessenspielraum eingeräumt ist, es also nicht nur um die Auslegung von sogenannten unbestimmten Rechtsbegriffen und die zuvorige Subsumierung des Tatbestandes unter dieselbigen geht, kann die Staatszielbestimmung zugunsten des Naturschutzes, der ja in der nationalen Rechtsordnung nirgends voraussetzungslos oder "absolut" geschützt ist, den Ausschlag geben. ${ }^{44}$ So entscheiden zum Beispiel die Naturschutzbehörden über Befreiungen von naturschutzrechtlichen Verboten und Geboten, wie sie sich aus § 31 BNatSchG und entsprechenden Bestimmungen der Gebietsschutzverordnungen ergeben, nach ihrem Ermessen. Hier wird die Staatszielbestimmung zur Erhaltung der natürlichen Lebensgrundlagen regelmäßig den Ausschlag für eine Entscheidung gegen den Befreiungsantrag geben, wenn etwa ein Naturschutzgebiet betroffen wäre.

$41 \quad$ Murswiek Kommentierung zu Art 20a GG Rn 68; Uhle 1996 UPR 56.

42 BVerwG NJW 1995, 2648.

$43 \quad$ Nach Murswiek 1996 NVwZ 225 ist das sogenannte "Gemeinlastprinzip ... als Prinzip der Zurechnung der Kosten von Umweltbelastungen grundsätzlich verfassungswidrig". 


\subsubsection{Staatszielbestimmungen als Abwägungshilfe}

In der Regel werden der verantwortlichen Behörde keine so weitgehende Handlungsspielräume (Entscheidungsprärogativen) eingeräumt wie bei echten Ermessenentscheidungen, die allerdings auch immer "pflichtgemäß", also zumindest nach entsprechender Sachverhaltsaufklärung zu treffen sind. Die Situation bei der sogenannten planerischen Abwägung ähnelt zwar der bei Ermessensentscheidungen, weil auch die Planungsbehörde regelmäßig "echte" und vom Verwaltungsgericht nur beschränkt überprüfbare Alternativen hat, die von den Juristen mit dem Kürzel "planerische Gestaltungsfreiheit" bezeichnet werden (früher: "Planungsermessen"). Allerdings sind die rechtlichen Bindungen so komplex und vielschichtig, daß eine vollständige Darstellung hier nicht möglich ist. Sehr vereinfacht läßt sich allerdings resümieren, daß bei derartigen Abwägungsvorgängen den Naturschutzbelangen heute ein stärkeres Gewicht zukommt als vor der Grundgesetzänderung. ${ }^{45}$ Der Schutzauftrag des Art. 20a GG kann als sogenannte Optimierungsgebot verstanden werden. ${ }^{46}$ Dies führt allerdings per se weder zu einem regelmäßigen noch zu einem tendenziellen Überwiegen der Naturschutzbelange. Ein entsprechendes Problem stellt sich im Rahmen der erforderlichen Abwägung zwischen Naturschutzbelangen und sonstigen Anforderungen an Natur und Landschaft im Rahmen der Eingriffs- und Ausgleichsregelung. ${ }^{47}$ Es ist dies die schwierige Frage nach dem verfassungsrechtlich verbindlichen Schutzniveau für den Naturschutz (und auch den Umweltschutz insgesamt). Neben einem Verschlechterungsverbot in bezug auf die Umweltsituation läßt sich aus Art. 20a GG ein "Integritätsmaßstab" ableiten, wonach für die heimischen Tiere und Pflanzen diejenigen Bedingungen erhalten bleiben oder wiederhergestellt werden müssen, die ihr Überleben als Art ermöglichen, und zwar in freier Natur und in heimischen Regionen. ${ }^{48}$ Darüber hinaus läßt sich der Verfassungsentscheidung noch entnehmen, daß eine Anhebung des Schutzniveaus anzustreben und umzusetzen ist, weil es der Dignität der Verfassung

44 Unklar Schink 1997 DÖV 228, der nicht deutlich zwischen Ermessensentscheidungen, Auslegung und "Gewichtungsdirektive" unterscheidet.

45 So auch Schink 1997 DÖV 228.

46 Murswiek Kommentierung zu Art 20a GG Rn 53 und 70.

47 Zur Eingriffs- und Ausgleichsregelung am Muster des bayerischen Landesrechts vgl Czybulka und Rodi Eingriffsregelung 513-525.

48 So ausdrücklich Murswiek 1996 NVwZ 226. 
widerspräche, wenn dem Volk nur "symbolische Politik" vorgemacht würde. ${ }^{49}$ Andererseits verbleibt es bei der "prinzipiellen Gleichordnung" des Umwelt- (und Natur)schutzes mit anderen Verfassungsprinzipien.

\section{$3 \quad$ Grundrechte und Naturschutz}

\subsection{Einführung}

Trotz bestehender Staatszielbestimmung ist das Thema "Grundrechte und Naturschutz" nicht obsolet geworden, weil Grundrechte als subjektiv-öffentliche Rechte (im Verfassungsrang) für den Bürger immer einen hohen rechtlichen Stellenwert haben. Nur bei Einräumung subjektiver Rechtspositionen hat der Bürger im gegenwärtigen (nationalen) Rechtssystem die Chance, notfalls auf dem (Verwaltungs-)Gerichtsweg "sein" Recht gegenüber dem Staat durchzusetzen. Wie unter 1.2 erwähnt, bietet der Komplex "Grundrechte und Naturschutz" erhebliche verfassungsrechtliche Probleme, wobei im Zentrum die Überlegung steht, daß die Beachtung der Menschenwürde (und nicht der Schutz der Natur) Maßstab aller verfassungsrechtlicher Wertentscheidungen der Verfassung sei (Art. 1 sowie Art. 79 Abs. 3 GG). Damit wird auch der Schutz der Natur, werden die "schützenswürdigen Interessen", an einer "menschlichen" Position festgemacht, die Anthropozentrik ist sozusagen verfassungsrechtlich unentrinnbar. ${ }^{52}$ Zum Teil wird wohl nach wie vor die Würde des Menschen in Opposition zur Physik (und Ökologie) der außermenschlichen Natur definiert. ${ }^{53}$ Vorgelagert ist dieser Problematik die grundsätzliche Frage, inwiefern Grundrechte (oder Rechte allgemein) auch Tieren und Pflanzen (außermenschlichem Leben) oder eventuell sogar Ökosystemen, sonstigen Arten-und Lebensgemeinschaften oder auch unbelebten Gegenständen zukommen können. In der Grundsatz-Diskussion über "Eigenrechte der Natur" wird oft übersehen, daß Grundrechte keine einheitliche Struktur haben, sondern

49 Murswiek 1996 NVwZ 226.

50 Gemeinsame Verfassungskommission, BT-Drs. 12/6000 66f.

51 Vgl Kloepfer Umweltschutz als Staatspflicht 313.

52 Hofmann $1988 \mathrm{JZ} 277$.

53 Vgl Hofmann 1988 JZ 269, der aber gerade dies ablehnt, weil es infolge des immanenten

Funktionszusammenhanges alles Lebendigen (und aus anderen Gründen) keinen Sinn mehr mache. 
vielgestaltig und auch in sich mehrdimensional sind. Schließlich setzen die Grundrechte nach derzeitigem Verständnis dem Naturschutz auch Grenzen (unten 3.6).

\subsection{Eigenrechte oder Grundrechte der Natur?}

Von der Rechtsprechung in Deutschland (Klage der "Seehunde der Nordsee"), aber auch von der ganz überwiegenden Meinung wird die Lehre von der möglichen Rechtsfähigkeit natürlicher Objekte abgelehnt. Das Verwaltungsgericht Hamburg hat bei der "Robbenklage" ausdrücklich darauf abgestellt, ${ }^{54} \mathrm{daß}$ tragender Grund für die (alleinige) Rechtssubjektivität des Menschen sei, daß nur ihm "die besondere Personenwürde eigen ist kraft seines Geistes", der inn von der unpersönlichen Natur abhebe. Auf der Gegenseite wird vehement gefordert, daß nur die Einräumung einer Rechtssubjektivität der Natur die Gleichwertigkeit zwischen Mensch und Natur herstellen könne. ${ }^{55}$ Kronzeuge für diese Auffassung dürfte Stone mit seiner aus dem anglo-amerikanischen Rechtskreis stammenden Streitschrift "Should Trees have Standing" aus dem Jahre 1972 sein. Die (auch) verfassungspolitische Diskussion über Eigenrechte hat einige Bedeutung für den Tierschutz und die Änderung der Vorschriften des Bürgerlichen Gesetzbuches ( $\$ 90 \mathrm{a}$ BGB) über Tiere gehabt, und sie ist sicherlich befruchtend für die (normative) Ethik gewesen. Anderseits läßt sich konstatieren, daß jene Denkweisen in das (deutsche) Verfassungsrecht keinen Eingang gefunden haben.

Es wird argumentiert, daß die Einräumung einer Subjektstellung der Natur und eine damit verbundene ganzheitliche oder "holistische" Denkweise zu anderen Rechtsüberzeugungen führe als die Betrachtung der Natur als "Antipode", als Objekt vor allem menschlicher Ausbeutung, jedenfalls als Objekt im Sinne eines anthropozentrischmechanistischen Weltbildes. $^{57}$ Als Zwischenstufe ist eine "geläuterte Anthropozentrik" denkbar. Dogmatisch plausibler erscheint der Vorschlag, die (Eigen-)Rechte der Natur als Schranken der Freiheitsrechte der Menschen zu verstehen. ${ }^{58}$ Die Diskussion darüber, welchen Teilen der Natur denn Eigenrechte zukommen sollten, wird zum Teil ausufernd

\footnotetext{
54 VG Hamburg, Beschluß v 22.09.1988 NVWZ 1058 f - Robbenklage.

55 Bosselmann 1987 NuR 4.

56 Von der Pfordten Ökologische Ethik.

57 Übersicht bei Heinz 1990 Der Staat $416 \mathrm{ff}$.

58 Weber 1991 IUR 85.
} 
geführt. Die Gegner einer Rechtssubjektivität der Natur führen hier zum Beispiel Bakterienstämme oder Viren auf, die wohl besser keine Rechtssubjektivität erhielten. Welchen bestimmten Tier- und Pflanzenarten, ganzen Ökosystem oder anderen Naturgütern Rechtssubjektivität zuerkannt würde, ist juristisch wohl nicht entscheidend. Es kommt darauf an, wer die Eigenrechte definiert und wer sie wahrnimmt ${ }^{60}$ beziehungsweise notfalls prozessual durchsetzen kann. Die Definition von Rechten der Natur und ihre Interessenzuteilung kann in einem Rechtssystem wieder nur der Mensch leisten, der als "Treuhänder" der Natur auftreten kann, wobei verschiedene Konstruktionsmöglichkeiten dieser Interessenvertretung denkbar sind. Bei der Feststellung und "Zuteilung" der Interessen der betroffenen Naturschöpfungen kommen diese selbst nur in Ausnahmefällen in Betracht, da innen zumeist die Kommunikationsfähigkeit im Rechtssinne fehlt, die regelmäßig an der Sprachbeherrschung ansetzt. Ausnahmen sind im Bereich des Tierschutzes bei höheren Tieren denkbar, die - vielleicht - keines "Dolmetschers" bedürfen. Gleichwohl bedarf es in aller Regel der "autoritativen" Feststellung eines Menschen (oder eines aus Menschen gebildeten "Organs" im Rechtssinne), um zum Beispiel den Eintritt einer Rechtsverletzung tatbestandlich zweifelsfrei feststellen zu können. Im Bereich des Naturschutzes sind infolge der komplexen ökologischen Zusammenhänge regelmäßig sachverständige Aussagen erforderlich. Zur Schaffung von Abhilfe bei naturschädigenden Zuständen ist wohl nur der Mensch in der Lage, und sei es nur dadurch, daß ein Veränderungsverbot ausgesprochen und durchgesetzt wird.

Tatsächlich werden die Interessen der betroffenen "natürlichen Lebensgrundlagen" rechtlich derzeit vor allem durch die öffentliche Verwaltung (hauptamtlicher Naturschutz) in Form von Einzelakten und Verordnungen wahrgenommen. Auch der Gesetzgeber nimmt diese Aufgabe seit einiger Zeit unmittelbar wahr, indem er bestimmte Biotope unter (gesetzlichen) Schutz stellt, ${ }^{61}$ und die zum Teil wesentlich detaillierteren Kataloge der Landesnaturschutzgesetze zum Biotopschutz. Da es für diese Unterschutzstellung keines weiteren Vollzugsaktes bedarf, liegt hierin bereits eine Zuteilung rechtlicher Interessen zum Beispiel an "Moore" oder "Salzwiesen und

59 Weitere Beispiele bei Weber 1991 IUR 81.

60 Letztlich natürlich auch noch, wie er sie wahrnimmt. Unbestreitbar ist im Naturschutzrecht das Vollzugsdefizit groß, vgl Czybulka 1996 JZ 599.

$61 \mathrm{Vgl} \S 20 \mathrm{c}$ BNatSchG. 
Wattflächen im Küstenbereich". Die Anhänge I-IV der FFH-Richtlinie können gleichfalls als "Interessenzuteilung" im Rechtssinne gewertet werden, insoweit sie zur (obligatorischen) Ausweisung von besonderen Schutzgebieten führen. Hier kommt hinzu, daß die Europäische Kommission die Umsetzung von Richtlinien in nationales Recht überwacht und nach Durchführung eines Vorverfahrens Vertragsverletzungsverfahren vor dem Europäischen Gerichtshof einleiten kann (Art. 169 EGV), so daß es hier im Prinzip auch eine gerichtliche Durchsetzung der Interessen der Natur gibt, weil sich der Rechtsschutz hier nicht aus subjektiven oder "Eigenrechten" der Natur ableitet.

Eine weitere Form der Interessenwahrnehmung der Rechte der Natur stellt im geltenden Recht die gemäß $\S 29$ BNatSchG - allerdings nur punktuell - vorgesehene Mitwirkung der anerkannten Naturschutzverbände (Der nichtstaatliche Naturschutz) und das oben im Abschnitt 2.2.3 erwähnte, in der Mehrzahl der Bundesländer existierende Verbandsklagerecht dieser Organisationen dar. Eine Stärkung dieser Wahrnehmungsrechte wäre verfassungsrechtlich sicherlich unbedenklich, angesichts der zunehmenden Naturzerstörung sogar ratsam. Art 20a GG vermittelt aber selbst keine Klagebefugnis, ${ }^{62}$ so daß die Länder handeln müßten. Denkbar wären hier auch andere Lösungen, zum Beispiel die Institutionalisierung eines Naturschutz-Ombudsmannes ${ }^{63}$ oder -Anwalts mit entsprechenden Befugnissen und Sachmitteln.

\subsection{Naturschutz durch "Umweltgrundrechte"?}

\subsubsection{Grundrechtliche Abwehransprüche gegen Umweltbelastungen und Naturzerstörung}

Art. 39 Abs. 2 der Verfassung des Landes Brandenburg enthält die Bestimmung:

"Jeder hat das Recht auf Schutz seiner Unversehrtheit vor Verletzungen und unzumutbaren Gefährdungen, die aus Veränderungen der natürlichen Lebensgrundlagen entstehen."

62 Murswiek Kommentierung zu Art 20a GG Rn 73.

63 Heinz 1994 NuR 8. 
Vorstehende Bestimmung ist in klassischer Lesart zunächst als Abwehrrecht des Bürgers gegen hoheitliche Beeinträchtigungen seiner Freiheitssphäre zu verstehen. Das Abwehrgrundrecht wäre im Idealfall gleichsam die subjektiv-individuelle Kehrseite der objektiven Verpflichtung des Staates, die natürlichen Lebensgrundlagen zu schützen. Die Etablierung eines derartigen Abwehrgrundrechts bringt jedoch schon allgemein im Umweltschutzbereich und speziell für den engeren Bereich des Naturschutzes nur einen begrenzten Ertrag. Zunächst richtet es sich unmittelbar nur gegen Naturbeeinträchtigungen, die durch den Staat beziehungsweise öffentlich-rechtliche Körperschaften selbst verursacht werden. Dies ist nur ein relativ kleiner Teil der Vorhaben, würde aber immerhin zahlreiche öffentliche Planungen, etwa von Infrastrukturmaßnahmen (Verkehrserschließung durch den Staat) betreffen. Soweit Umwelt- und Naturgefährdungen von Privaten hervorgerufen werden - das dürfte die Mehrzahl der Eingriffe etwa durch Überbauung und Gewerbe sein - entfalten die Grundrechte allenfalls mittelbare Wirkung. ${ }^{64}$ Soweit von der Rechtsprechung des Bundesverfassungsgerichts $^{65}$ und der Lehre ${ }^{66}$ weitergehende Schutzpflichten des Staates gegenüber rechtswidrigen Eingriffen Dritter abgeleitet werden, betrifft dies zunächst typische Gefährdungstatbestände, etwa - wie hier - der körperlichen Unversehrtheit des Menschen. Die Verarmung der Natur, der Rückgang der Artenvielfalt und der ästhetischen Qualitäten der Natur sind aber nach herrschendem juristischen Verständnis keine Gefahren für die Gesundheit, die hier unter die Schutzpflicht des Staates gestellt werden könnten. Außerdem kann nach der Rechtsprechung des Bundesverfassungsgericht eine Schutzpflichtverletzung des Staates nur in extremen Fällen angenommen werden, etwa bei gänzlicher Untätigkeit der staatlichen Organe oder evidenter Unzulänglichkeit der bisherigen Maßnahmen. Für den Naturschutz fehlt dem Bürger auch im einfachen Recht regelmäßig jede anspruchsbegründende Zuordnung, die etwa durch das Grundrecht verstärkt werden könnte. Die Natur wird insoweit nämlich "um ihrer selbst willen geschützt". Nach allgemeiner Auffassung hat im deutschen öffentlichen Recht kein Bürger den Anspruch auf Ausweisung und/oder Erhaltung eines

64 Schmidt und Sandner Einführung in das Umweltrecht 71.

65 Vgl etwa BVerfGE 88, 203, 251 zum Schutz des (ungeborenen) menschlichen Lebens.

66 Vgl Jeand'Heur $1995 \mathrm{JZ} 161 \mathrm{ff}$. 
Schutzgebietes, ${ }^{67}$ eines Biotopes oder einer sonstigen, natürlichen (oder naturnäheren) Lebensgemeinschaft oder "Situation". Die Normen sind insoweit nicht "drittschützend". ${ }^{68}$ Art. 20a GG bewirkt also auch keinen Drittschutz. Lediglich in Bezug auf schwere Beeinträchtigungen von Betrieben, die von einer einigermaßen intakten Umwelt abhängen (zum Beispiel Berufsfischer), hat das Bundesverwaltungsgericht aus dem Gesichtspunkt des Eigentumsschutzes nach Art. 14 GG einen Drittschutz prinzipiell bejaht, wenn der Bestand des Gewerbebetriebs ernsthaft in Frage gestellt wird. ${ }^{69}$ Den "Abschied von der Schutznormtheorie im Natur- und Landschaftsschutz" hat Sening schon 1980 gefordert, allerdings bislang ohne durchschlagenden Erfolg.

\subsubsection{Grundrecht auf intakte Natur?}

Der Ausweg scheint zunächst naheliegend, dem einzelnen Menschen unmittelbar ein (positives) "Umweltgrundrecht" beziehungsweise ein "Grundrecht auf intakte Umwelt" einzuräumen, das dann zugleich ein Grundrecht auf intakte Natur beinhaltete. Ein derartiges Grundrecht hätte eine im Vordergrund stehende Leistungskomponente; es ließe sich als positiver "Anspruch" des einzelnen auffassen. An eine derartige Konstruktion hat sich aber kein Verfassungsgeber herangewagt. Allgemein wird hier kritisiert, ein derartiges Grundrecht auf intakte Umwelt beziehungsweise Natur sei in Anbetracht der bestehenden Umweltschäden unerfüllbar; es würden hiermit nur unrealisierbare Hoffnungen geweckt. ${ }^{71}$ Dem ist im Ergebnis, wenn auch nicht ganz im Ansatz zuzustimmen, weil Grundrechte zumeist auch "kontrafaktische" Elemente enthalten. Allerdings fügte sich ein derartiges Grundrecht nicht in das bestehende Grundrechtssystem ein. Ein erster Schritt könnte verfassungspolitisch gesehen - die Anerkennung eines ökologischen Existenzminimums ${ }^{72}$ als Grundrecht sein, das sich dogmatisch an das - anerkannte - Recht auf ein ökonomisches (und damit auch soziales)

67 Denkbar wäre als Ausnahme, daß zB ein Naturschutzverband Eigentümer einer unter Natur-schutz gestellten Fläche ist. Hier resultieren die Abwehrrechte dann aber aus Art 14 GG (Eigentum!).

68 Czybulka Rechtspflichten des Bundes 40.

69 Vgl BVerwG DÖV 1983342 f: ... Genehmigte Einleitung von Dünnsäure führt zu erheblich reduzierten Fangerträgen ...

70 Auf den Zusammenhang von Kontrolldefiziten und Schutznormtheorie macht Heinz 1990 Der Staat 435 m.w.Nw., aufmerksam.

71 Schmidt und Sandner Einführung in das Umweltrecht 72.

72 Begriff wohl von Scholz 1976 JuS 234. 
Existenzminimum anlehnt, ${ }^{73}$ aber nicht ausschließlich aus dem Menschenwürde-Postulat abgeleitet wird, sondern insoweit mehrdimensional sein müßte. Das ökologische Existenzminimum könnte als wesentlichen Teilinhalt den Anspruch auf Erhaltung und Schutz der naturnahen Lebensräume der heimischen Tier-und Pflanzenarten einschließlich eines maßvollen Erholungsanspruchs enthalten. Perspektivisch sollte vor allem auf die Bewahrung der Natur "für künftige Generationen" abgestellt werden.

\subsubsection{Recht auf Naturgenuß}

In der Bayerischen Verfassung ist in Art. 141 Abs. 3 S. 1 ein Grundrecht auf Genuß der Naturschönheiten und auf Erholung in der freien Natur verankert. ${ }^{74}$ Es ist schon vom Ansatz problematisch, ob der Schutz der Natur durch eine derartige, (extrem) anthropozentrische Verfassungsnorm gesichert werden könnte. Wie bereits dargelegt (oben 2.2.3), hat die Rechtsprechung den Gehalt der Norm inzwischen erheblich reduziert. ${ }^{75}$

\subsection{Naturschutz als sogenannte "Grundpflicht"}

Neben Grundrechten kennen die Verfassungen auch sogenannte "Grundpflichten", ${ }^{76}$ etwa die Wehrpflicht (Art. 12a GG), aber auch die Pflicht, Steuern zu zahlen. In mehreren Landesverfassungen finden sich im Zusammenhang mit dem Schutz der natürlichen Lebensgrundlagen Formulierungen, die an eine derartige "Grundpflicht" denken lassen. So heißt es etwa in der Bayerischen Verfassung als Anhang zum "Recht auf Naturgenuß" in Art. 141 Abs. 3 BV:

"Dabei ist jedermann verpflichtet, mit Natur und Landschaft pfleglich umzugehen."

In Art. 39 Abs. 1 der Landesverfassung von Brandenburg heißt es:

73 Vgl BVerfGE 82, 60, 85, 87, 153, 173 ff; BVerfG NJW 1994, 991; weiterführend Neumann 1995 NVWZ 426-432.

74 Ähnlich SachsVerf Art 10 Abs 3.

75 Vgl dazu näher Soell 1986 WiVerwW $213 \mathrm{ff}$.

76 Vgl hierzu Götz und Hofmann Grundpflichten $7 \mathrm{ff}, 42 \mathrm{ff}$. 
"Der Schutz der Natur, der Umwelt und der gewachsenen Kulturlandschaft als Grundlage gegenwärtigen und künftigen Lebens ist Pflicht des Landes und aller Menschen."

Die wichtigste verfassungsrechtliche Pflicht im Bereich des Naturschutzes ist die Sozialbeziehungsweise Ökologiepflichtigkeit des Eigentums, die sich aus den diesbezüglichen Inhalts- und Schrankenbestimmungen ergibt (unten 3.5.1). Es wird nicht recht deutlich, welche weiteren "Pflichten" den Bürgern durch diese besondere Herausstellung in der Verfassung auferlegt werden sollen. Denkbar wäre etwa eine weitergehende Beschränkung des Betretungsrechts, ${ }^{78}$ der Verschmutzung oder Verlärmung der Natur, aber auch ein bloß appellativer Charakter der Vorschrift.

\subsection{Naturschutz als Schranke der Grundrechtsbetätigung}

\subsubsection{Sozialpflichtigkeit beziehungsweise Ökologiepflichtigkeit des Eigentums}

Die Staatszielbestimmung des Schutzes der natürlichen Lebensgrundlagen muß in eine vernünftige Beziehung zur Eigentumsgarantie des Art. 14 GG gesetzt werden, sonst "taugt sie wenig". ${ }^{79}$ Obwohl die verfassungsrechtliche Gewährleistung des Eigentums für die öffentliche Hand nicht gilt, ${ }^{80}$ gilt diese Pflichtendimension natürlich auch für sie. Trotzdem wird derzeit (1998) mit dem Verkauf von "Fiskalvermögen" zum Beispiel in Nationalparken die Staatskasse aufgebessert, ein Verstoß gegen die Vorbildfunktion des Staates im Bereich des Naturschutzes. Der Eigentumsschutz privater Naturnutzer und belaster, vor allem der Land- und Forstwirte, ist verfassungsrechtlich mehrfach beschränkbar. Dies betrifft zunächst etwa das Grundwasser und den Wasserhaushalt, der einer öffentlich-rechtlichen Benutzungsordnung unterworfen ist, die die Privatnützigkeit auch völlig aufheben kann. ${ }^{81}$ Daneben hat der Gesetzgeber weitgehende Möglichkeiten,

77 Vertiefend Führ 1998 NuR 6-14.

78 Dies diskutiert Soell 1986 WiVerwW in Bezug auf BayVerf Art 141 Abs 3 S 212.

79 Hofmann Technik und Umwelt 1038.

80 Vgl BVerfG NJW 19901783.

81 Vgl BVerfGE 58, 300 - Naßauskiesungsbeschluß. 
"Inhalt und Schranken" des Eigentums zu bestimmen, etwa bei der Bodennutzung. Das Grundeigentum wird geprägt durch seine "Situationsgebundenheit", was im Außenbereich dazu führt, daß naturschutzrechtliche Nutzungsbeschränkungen und untersagungen in der Regel entschädigungslos hinzunehmen sind. Dies gilt auch für Nutzungen, die zuvor rechtlich zulässig waren, wenn sie längere Zeit nicht ausgeübt wurden. Auch ausgeübte, zulässige Nutzungen können im Rahmen der "Sozialpflichtigkeit" des Eigentums, die sich hier als "Ökologiepflichtigkeit" darstellt, beschnitten werden. "Ausgleichspflichtig" wird eine solche Inhaltsbestimmung erst, wenn von dem Betreffenden im Verhältnis zu anderen ein "Sonderopfer" verlangt wird, das eine erhebliche Belastung darstellen muß. Maßstab ist nicht etwa der profitmaximierende industrialisierte Betrieb, sondern der einsichtige Eigentümer beziehungsweise Landwirt. Die Einschränkung etwa der Anwendung von Kunstdünger oder Gülle ist verfassungsrechtlich problemlos. ${ }^{84}$ Trotz dieser für den Naturschutz an sich günstigen verfassungsrechtlichen Ausgangslage und der tendenziell "naturschutzfreundlichen" Rechtsprechung fehlt es weitgehend am politischen Willen, diese verfassungsrechtlichen Möglichkeiten zugunsten des Naturschutzes durchzusetzen. Obwohl die für den Naturschutz wertvolleren Flächen oftmals ohnehin keine intensive landwirtschaftliche Nutzung zulassen, ihre Situationsgebundenheit also ihren Verkehrswert mindert, werden im Wege freiwillig gezahlter Billigkeitsentschädigung oder durch vertragliche Regelungen unterhalb der verfassungsrechtlich gebotenen Entschädigungs-pflicht Zahlungen an die Landwirte geleistet. Dies mag ökonomisch sinnvoll sein, wird aber möglicherweise auf Dauer die Rechtsprechung beeinflussen. Der "weichen Linie" durch sogenannten Vertragsnaturschutz entspricht es, wenn auch für den Naturschutz besonders wertvolle Flächen in der Praxis kaum jemals enteignet werden, obwohl dies das Grundgesetz (und alle Landesnaturschutzgesetze) zulassen. Hierfür wäre allerdings volle Entschädigung (Verkehrswert) zu leisten. Der Gesetzgeber, der ja vorrangig Adressat des Art. 14 Abs. 1 S. 2 GG ist, hat es seit Jahrzehnten nicht fertiggebracht, die Landwirtschaftsklauseln im Naturschutzrecht zu bereinigen, die nicht

85 Vgl etwa BVerwGE 84, 361 - Serriesteiche; und BVerwGE 94, 1 - Herrschinger Moos. 
nur eine gesetzgeberische Fiktion, sondern sogar europarechtswidrig, ${ }^{86}$ möglicherweise seit Einführung des Art. 20a GG sogar verfassungswidrig sind. Für die Land- und Forstwirte wären dynamische "Betreiberpflichten" gesetzlich festzuschreiben, die inhaltliche Maßstäbe einer ordnungsgemäßen und naturschonenden landwirtschaftlichen Bodennutzung festlegen. ${ }^{87}$ Regelungen über die Grenzen der Sozial- beziehungsweise Ökologiepflichtigkeit müßten auch berücksichtigen, daß der eingebrachte (rechtmäßige) Aufwand wohl den wichtigsten Parameter für eine verfassungsrechtliche Garantie des Eigentums an Natur darstellt. $^{88}$

3.5.2 Die Staatszielbestimmung des Art. 20a GG als ökologische Grundrechtsschranke

Einige Grundrechte sind in der Verfassung "voraussetzungslos" gewährt, sie unterliegen keinem ausdrücklichen Gesetzesvorbehalt. Sie können also grundsätzlich nicht durch Gesetz eingeschränkt werden. Dies gilt etwa für die Wissenschafts- und die Kunstfreiheit. Durch die Einfügung des Art. 20a GG kann dieser jetzt als sogenannte "verfassungsimmanente Schranke" gesetzliche Eingriffe in diese Grundrechte legitimieren. Das Bundesverfassungsgericht hat diese Argumentation bei Tierversuchsbeschränkungen ${ }^{90}$ und zur Einschränkung der Kunstfreiheit ${ }^{91}$ schon verwendet.

\subsection{Grundrechtliche Grenzen des Naturschutzes}

Gegenläufig zu den Überlegungen unter 3.3 bis 3.5.2 genießen in gewissem Umfang allerdings auch Umweltbelaster beziehungsweise Naturzerstörer Grundrechtsschutz, ${ }^{92}$ wobei vor allem Art. 14 (Eigentum) und Art. 12 GG (Berufs- und Gewerbefreiheit) zur Verfügung stehen, für bloße Freizeit- und sonstige Betätigungen auch die allgemeine Handlungsfreiheit des Art. 2 Abs. 1 GG. Diese wohl herrschende Auffassung entspricht

86 EuGH, Urteil vom 17.09.1987, 1988 NuR 53 zu § 22 Abs 3 BNatSchG früherer Fassung § 20 f Abs 3 BNatSchG aF.

87 Vgl Czybulka 1997 AgrarR 305.

88 In diese Richtung Heinz 1994 NuR 3.

89 Ausdruck nach Kuhlmann 1995 NuR 1, 9.

90 BVerfG 1994 NVWZ 894.

91 BVerwG 1995 NJW 2648. 
dem herkömmlichen liberalen Grundrechtsverständnis und seiner Schrankendogmatik. Allerdings wird im Schrifttum die These vertreten, eine "allgemeine Umweltverschmutzungsfreiheit" könne es nicht geben. Murswiek begründet seine These, mit der er explizit den Boden des liberalen Grundrechtsverständnisses nicht verlassen will, mit der "Voraussetzungshaftigkeit" der (wirtschaftlichen) Betätigungsfreiheit, die (jedenfalls) nicht den Zugriff auf Rechtsgüter Dritter (mit) gewährleiste. Dies gelte für den Zugriff auf das Eigentum Dritter unstreitig, sei aber auch maßgeblich für den Zugriff auf Gemeinschaftsgüter. Die Belastung der Gemeinschaftsgüter Luft, Wasser und Boden, zu denen auch die Folgewirkungen unter andere auf Tiere oder Pflanzen gezählt werden können, sei nicht lediglich Freiheitsausübung, sondern Teilhabe. Diese aber müsse verfassungsrechtlich ausdrücklich gewährleistet oder wenigstens ableitbar sein. Dies sei jedenfalls für die Belastung mit Schadstoffen oder mit Abfällen im industriellen Maßstab nicht der Fall. Die Verfassung gewährleiste nicht etwa die Umweltverschmutzung als umfassend (wenn auch gesetzlich einschränkbar), sondern (nur) das für die menschliche Existenz notwendige Minimum an Umweltverschmutzung werde gewährleistet. Die Übertragung dieser interessanten Gedanken auf den Bereich des verfassungsrechtlichen Naturschutzes steht noch aus. Immerhin hat das Bundesverfassungsgericht ein Besitz-, Verarbeitungs- und Vertriebsverbot für lebende und tote Vögel der besonders geschützten Arten noch vor Inkrafttreten des Art. 20a GG deshalb (im wesentlichen) für verfassungsgemäß erachtet, weil solche Tiere ...

..."Bestandteil des Naturhaushalts [sind], der durch mannigfache Faktoren zunehmend gefährdet ist und dessen Erhaltung im hohen Maße -auch im Interesse künftiger Generationen - dem Gemeinwohl dient.".

93 Hofmann Technik und Umwelt $1022 f$.

93 Murswiek Privater Nutzen und Gemeinwohl $79 \mathrm{ff}$

94 BVerfGE 61, 291, 307 - Tierpräparatorienentscheidung 


\section{LITERATURVERZEICHNIS}

Bosselmann 1987 NuR 1-6

Bosselmann K "Die Natur im Umweltrecht - Plädoyer für ein ökologisches

Umweltrecht" 1987 NuR 1-6

Czybulka 1988 NuR 214-220

Czybulka D "Eigentum an Natur" 1988 NuR 214-220

Czybulka 1996 NuR 565-570

Czybulka D "Rechtliche Möglichkeiten der Mitwirkung des Bundes bei Ausweisung,

Entwicklung und Management von Nationalparken in der Bundesrepublik

Deutschland" 1996 NuR 565-570

Czybulka 1996 JZ 596-602

Czybulka D "Umweltschutzdefizite und Verwaltungskultur" 1996 JZ 596-602

Czybulka Rechtspflichten des Bundes 39-53

Czybulka D "Rechtspflichten des Bundes und der Länder zur Ausweisung und

Beibehaltung von Schutzgebieten und Biotopen" in Naturschutzrecht und

Landschaftsplanung in europäischer Perspektive (Nomos-Verlaganstalt Baden-Baden 1996) 39-53

Czybulka 1997 AgrarR 305-310

Czybulka D "Naturschutzrechtlicher Flächen-und Artenschutz und landwirtschaft-liche Produktion" 1997 AgrarR 305-310

Czybulka und Rodi Bayerischen Naturschutzgesetz 513-525

Czybulka D und Rodi K Die Eingriffsregelung im Bayerischen Naturschutzgesetz BayVBI 1996 513-525 
Führ 1998 NuR 6-14

Führ M "Ökologische Grundpflichten als verfassungsrechtliche Dimension" 1998 NuR 6-14

Götz und Hofmann Grundpflichten 7 ff 42 ff Götz V und Hofmann H "Grundpflichten als verfassungsrechtliche Dimension: Berichte und Diskussionen auf der Tagung der Vereinigung der Deutschen Staatsrechtslehrer in Konstanz vom 6. bis 9. Oktober 1982" in Wahl und Pietzcker Verwaltungsverfahren zwischen Verwaltungseffizienz und Rechtsschutzauftrag (De Gruyter Berlin 1924-) VVDStRL 1983417 ff 42 ff

Heinz 1994 NuR 1-8 Heinz E "Staatsziel Umweltschutz in rechtstheoretischer und verfassungstheoretischer Sicht" 1994 NuR 1-8

Heinz 1990 Der Staat 415-439 Heinz K "Eigenrechte der Natur" 1990 Der Staat 415-439

Henneke 1995 NuR 325-335 Henneke H-G "Der Schutz der natürlichen Lebensgrundlagen in Art 20a GG: inhalt und Wirkungen einer ausbalancierten Staatszielbestimmung" 1995 NuR 325-335

Hofmann 1988 JZ 265-278 Hofmann H "Natur und Naturschutz im Spiegel des Verfassungsrechts" $1988 \mathrm{JZ}$ 265-278

Hofmann Technik und Umwelt 1005-1038 Hofmann $\mathrm{H}$ "§ 21: Technik und Umwelt" in Maihofer ea (Hrsg) Handbuch des Verfassungsrechts der Bundesrepublik Deutschland 2. Aufl (De Gruyter Berlin 1995) 1005-1038 
Jeand'Heur 1995 JZ 161-167

Jeand'Heur B "Grundrechte im Spannungsverhältnis zwischen subjektiven Freiheitsgarantien und objektiven Grundsatznormen" 1995 JZ 161-167

Kloepfer Umweltschutz als Staatspflich 305-316

Kloepfer M Umweltschutz und Verfassungsrecht -Zum Umweltschutz als Staatspflicht DVBI 1988 305-316

Kuhlmann 1995 NuR 1-10 Kuhlmann H "Der Mitweltschutz im gesamtdeutschen Grundgesetz" 1995 NuR 110

Ladeur 1994 NuR 8-14

Ladeur K-H "Berufsfreiheit und Eigentum als verfassungsrechtliche Grenze der staatlichen Kontrolle von Pflanzenschutzmitteln und Chemikalien" 1994 NuR 814

Murswiek Privater Nutzen und Gemeinwohl 76-88 Murswiek D Privater Nutzen und Gemeinwohl im Umweltrecht DVBI 1994 76-88

Murswiek Kommentierung zu Art 20a GG 653-668 Murswiek D "Kommentierung zu Art 20a GG" in Sachs M (Hrsg) Grundgesetz Kommentar (Beck München 1996) 653-668

Murswiek 1996 NVWZ 222-230 Murswiek D "Staatsziel Umweltschutz (Art. 20a GG): Bedeutung für Rechtsetzung und Rechtsanwendung" 1996 NVWZ 222-230

Nida-Rümelin und Von der Pfordten Ökologische Ethik Nida-Rümelin J und Von der Pfordten D (Hrsg) Ökologische Ethik und Rechtstheorie (Nomos-Verlaganstalt Baden-Baden 1995) 
Niesslein Naturschutz und Industriegesellschaft $45 \mathrm{ff}$

Niesslein E Naturschutz und Industriegesellschaft: Vorschlaege fuer eine neue Politik (Nomos-Verlagsanstalt Baden-Baden 1992) $45 \mathrm{ff}$

Neumann 1995 NVwZ 426-432

Neumann V "Menschenwürde und Existenzminimum" 1995 NVWZ 426-432

Peters 1995 NVWZ 555-557

Peters H-J "Art. 20a GG -Die neue Staatszielbestimmung des Grundgesetzes" 1995 NVWZ 555-557

Schink 1997 DÖV 221-229

Schink A "Umweltschutz als Staatsziel" 1997 DÖV 221-229

Schmidt und Sandner Einführung in das Umweltrecht 67-90 Schmidt R und Sandner W "Einführung in das Umweltrecht. Rechtsquellen/ Prinzipien, Instrumente des Umweltrechts" in Stengel M und Wüstner K (Hrsg) Umweltökonomie eine interdisziplinaere Einfuehrung (Vahlen Muenchen 1997) 67-90

Scholz 1976 JuS 232-237

Scholz R "Nichtraucher contra Raucher" 1976 JuS 232-237

Sening 1980 NuR 102-110

Sening $\mathrm{CH}$ "Abschied von der Schutznormtheorie im Naturschutzrecht" 1980 NuR $102-110$

Soell 1986 WiVerwW 205-223

Soell H "Der mediale Umweltschutz im geltenden Verfassungsrecht" 1986 WiVerwW 205-223 
Stone 1972 Southern California Law Review $450 \mathrm{ff}$

Stone CD "Should Trees Have Standing" in Toward Legal Rights for Natural Objects 1972 Southern California Law Review $450 \mathrm{ff}$

Tsai Umweltschutzpflicht des Staates 48 ff Tsai T-J Die verfassungsrechtliche Umweltschutzpflicht des Staates: zugleich ein Beitrag zur Umweltschutzklausel des Art. 20a GG (Duncker und Humblot Berlin 1996)

Uhle 1996 UPR 55-57

Uhle A "Das Staatsziel 'Umweltschutz' und das Bundesverwaltungsgericht: Anmerkungen zu dem Beschluß vom 12.4.1995 - 4 B 70/95" 1996 UPR 55-57

Von der Pfordten Ökologische Ethik 567

Von der Pfordten D Ökologische Ethik, Zur Rechtfertigung menschlichen Verhaltens gegenüber der Natur in Rowohlts Enzyklopaedie (Rowohlt Reinbek bei Hamburg 1996) 567

Weber 1991 IUR 2 81-86

Weber J "Rechtsstaat und Rechte der Natur" 1991 IUR 2 81-86

Gesetzliste Bundesnaturschutzgesetz

Gesetz über Naturschutz und Landschaftspflege vom 20. Dezember 1976 (BGBI.I

1976, S. 3574 und 1977, S. 650) in der Fassung der Bekanntmachung vom 12.

März 1987 (BGBI.I S. 889) 


\section{ABKÜRZUNGEN}

$\begin{array}{ll}\text { Abs } & \\ \text { Abs } & \text { Abschnitt } \\ \text { AgrarR } & \begin{array}{l}\text { Agrarrecht Zeitschrift für das gesamte Recht der Landwirtschaft, der } \\ \text { Agrarmärkte und des ländlichen Raumes }\end{array} \\ \text { Art } & \text { - Artikel } \\ \text { BayVerwGH } & \text { - Bayerischer Verwaltungsgerichtshof } \\ \text { BayVGH } & \text { - Bayerischer Verfassungsgerichtshof } \\ \text { BNatSchG } & \text { - Bundesnaturschutzgesetz } \\ \text { BR-Drs } & \text { - Bundesrats-Drucksache } \\ \text { BT-Drs } & \text { - Bundestags-Drucksache } \\ \text { Nr } & \text { - Nummer } \\ \text { NuR } & \text {-Natur und Recht } \\ \text { NVwZ } & \text {-Neue Zeitschrift für Verwaltungsrecht } \\ \text { Rn } & \text { - Randnummer(n) } \\ \text { S } & \text { - Satz } \\ \text { UPR } & \text { - Umwelt- und Planungsrecht } \\ \text { UVPG } & \text { - Umweltverträglicheitsprüfung } \\ \text { VVDStRL } & \\ & \text {-Veröffentlichungen } \\ & \end{array}$

